\title{
Urabstimmung FMH Services
}

\begin{abstract}
Weshalb eine Urabstimmung?
Durch die Statutenänderung bei der Verbindung der Schweizer Ärztinnen und Ärzte FMH ist die Doppelmitgliedschaft mit der Genossenschaft FMH Services aufgehoben worden. Neu muss jedes FMH-Mitglied ausdrücklich die Aufnahme in die Genossenschaft verlangen. In der Ärztekammer, welche auch zusätzlich die Delegiertenversammlung der Genossenschaft FMH Services darstellt, könnten somit auch NichtGenossenschafter Einsitz haben. Es ist daher nicht mehr möglich, die Delegiertenversammlung der FMH Services zusammen mit der ordentlichen Ärztekammer durchzuführen. Die Verwaltung hat deshalb beschlossen, die ordentlichen Geschäfte sowie eine notwendige Statutenänderung direkt der Urabstimmung zu unterstellen.
\end{abstract}

\section{Wer ist stimmberechtigt?}

Stimmberechtigt sind alle FMH-Mitglieder, welche vor dem 31. Dezember 1999 in die FMH als ordentliches oder ausserordentliches Mitglied aufgenommen worden sind. Weiter alle FMH-Mitglieder, welche sich beim Eintritt in die FMH auch zum Beitritt in die Genossenschaft FMH Services entschlossen haben.

\section{Geschäftsbericht 1999}

Die Umstrukturierung der FMH Services wurde im vergangenen Jahr abgeschlossen. Die Genossenschaft ist nun als Holding strukturiert. Alle operativen Tätigkeiten werden durch spezielle Beteiligungsgesellschaften wahrgenommen. Diese Beteiligungsgesellschaften sind selbständige Aktiengesellschaften, deren Aktienmehrheit in den Händen der Holdinggenossenschaft liegt. Der Geschäftsführer der FMH Services ist in allen Gesellschaften im Verwaltungsrat vertreten. Er rapportiert zuhanden der Verwaltung über die Tätigkeiten der einzelnen Gesellschaften. Detaillierte Informationen über diese Tätigkeiten der Beteiligungsgesellschaften können den einzelnen Jahresberichten entnommen werden, welche ebenfalls in der Schweizerischen Ärztezeitung publiziert werden. Die Umgruppierung in eine Holdingstruktur lässt es auch zu, dass die Verwaltung verkleinert werden kann. Die um eine Person auf vier Personen verkleinerte Verwaltung kann ihre Aufgaben professionel-

\section{Wie wird abgestimmt?}

Alle Unterlagen für die Urabstimmung sind nachfolgend publiziert. Alle Anträge sind auf einem separaten Stimmzettel zusammengefasst. Der ausgefüllte Stimmzettel wird im Stimmcouvert in das beiliegende Rückanwortcouvert gesteckt und an die bezeichnete Treuhandstelle geschickt. Um gültig zu stimmen, ist es wichtig, das Rückantwortcouvert mit Praxisstempel und persönlicher Unterschrift zu versehen. Nur so kann gewährleistet werden, dass ausschliesslich Genossenschaftsmitglieder ihre Stimme abgeben.

\section{Welche Frist ist zu beachten?}

Die Stimmzettel müssen bis spätestens 10. August 2000 bei der Treuhandstelle eingetroffen sein.

\section{Wer erteilt Auskünfte?}

Für Fragen steht der Geschäftsleiter der FMH Services, Markus Baumgartner unter der Telefonnummer 0419250077 gerne zu Ihrer Verfügung.

Für die Verwaltung der FMH Services

Dr. med. H. H. Brunner

Dr. med. M. Giger Präsident ler wahrnehmen. Die Verwaltung schlägt eine entsprechende Statutenänderung vor. Da aufgrund der neuen FMH-Statuten die Mitgliedschaft zwischen FMH und FMH Services nicht mehr automatisch gekoppelt ist, müssen die Statuten zusätzlich den neuen Gegebenheiten angepasst werden. Die Verwaltung unterbreitet die entsprechenden Änderungen in der vorliegenden Urabstimmung allen Mitgliedern.

Der Jahresabschluss der FMH Services spiegelt die Umstrukturierung ebenfalls wider. Nach der Auflösung nicht benötigter Reserven, welche den FMH Services im Vorjahr einen Gewinn von Fr. 244 000.einbrachten, schliesst die Jahresrechnung mit einem Gewinn von Fr. 2653.- ab.

Auf Ende des Geschäftsjahres verlässt Dr. med. Eduard Eicher, Basel, die Verwaltung. Verwaltung und Geschäftsleitung möchten an dieser Stelle Dr. Eicher ihren Dank für die geleistete Arbeit aussprechen.

Im laufenden Geschäftsjahr wird die FMH Services Gruppe weiter alles daran setzen, die Palette der Dienstleistungen zusammen mit anderen Partnern im Gesundheitswesen auszubauen und die Genossenschafter tatkräftig zu unterstützen. 


\section{Bilanz per 31.12.1999}

Geschäftsjahr 1999

Bezeichnung

Aktiven

Umlaufvermögen

Banken

Wertschriften

Flüssige Mittel $u$. Wertschriften

Forderungen aus Leistungen gegenüber Dritten

Forderungen aus Leistungen gegenüber verb. Organisationen

Andere kurzfristige Forderungen gegenüber verb. Organisationen

Andere kurzfristige Forderungen

Total Forderungen

Aktive Rechnungsabgrenzung

Total Umlaufvermögen

\section{Anlagevermögen}

Anteilscheine

Beteiligungen

Langfristige Forderungen gegenüber Tochtergesellschaften

Total Finanzanlagen

Mobile Sachanlagen

Immobile Sachanlagen

Total Anlagevermögen

Total Aktiven
2238200.00

63802.00

1231800.00

3533802.00

3948228.98
107634.66

158263.20

265897.86

35890.45

12970.50

Fr. Bezeichnung

Fr.

Passiven

Fremdkapital kurzfristig

Kurzfristige Verbindlichkeiten

Kurzfristige Verbindlichkeiten ggn. Verb. Gesellschaften

36855.40

602.00

Kurzfristige Finanzverbindlichkeiten gg. verb. Organisationen

4706.10

Passive Rechnungsabgrenzung

Total Fremdkapital kurzfristig

20600.00

62763.50

Fremdkapital langfristig

Langfristige Finanzverbindlichkeiten $\quad 745000.00$

Langfristige Rückstellungen

129750.00

Total Fremdkapital langfristig

874750.00

1215.25

414426.98

Eigenkapital

Allgemeine Reserve

Bilanzgewinn

1000.00

617200.00

1620000.00

Total Eigenkapital

3008062.36

2653.12

$\underline{\text { Total Passiven }}$
3010715.48

3948228.98

\section{Erfolgsrechnung per 31.12.1999}

\section{Geschäftsjahr 1999}

Bezeichnung

Betriebsertrag

Aufwandminderungen

Bruttoergebnis 1

Personalaufwand

Bruttoergebnis 2

sonstiger Betriebsaufwand

Cash-flow

Abschreibungen

Betriebsergebnis 1

betriebliche Nebenerfolge

Betriebsergebnis 2

ausserordentlicher Erfolg

Unternehmenserfolg vor Steuern

Steuern

Unternehmensgewinn
Fr.

1559.15

0.00

1559.15

$-52684.00$

$-51124.85$

$-37972.88$

$-89097.73$

$-60638.70$

$-149736.43$

124475.15

$-25261.28$

37991.20

12729.92

$-10076.80$

$\underline{2653.12}$

Bericht der Kontrollstelle

Die Kontrollstelle PriceWaterhouseCoopers hat die Rechnung genehmigt und empfiehlt sie der Urabstimmung zur Annahme. 


\section{Anpassung der Statuten}

Die FMH-Services-Statuten* sind in diversen Artikeln anzupassen. Die angepassten Artikel werden in den Schlussbestimmungen aufgeführt. Anschliessend sind die neuen Artikel selbst aufgeführt:

\section{Schlussbestimmungen}

\section{Art. 31 Genehmigung durch die Delegierten- versammlung}

Diese Statuten sind von der Delegiertenversammlung am 20. Juni 1990 angenommen, am 20. Juni 1996 und 24. Juni 1998 bezüglich Art. 1 revidiert worden.

Art. 32 Statutenänderung durch die Urabstimmung Die Statuten wurden durch die Urabstimmung vom ...... revidiert hinsichtlich:

II. Mitgliedschaft, Art. 4, Absatz 1 und 2

III. Organisation des «FMH Services»; A. Die Gesamtheit der Mitglieder, Art. 9.b) und B. Delegiertenversammlung, Art. 11 und Art. 12 sowie C. Verwaltung, Art. 16

\section{Neufassung der eingangs erwähnten Artikel}

\section{A. Die Gesamtheit der Mitglieder}

Art. 9 Gegenstand

In einer Urabstimmung entscheidet die Gesamtheit der Mitglieder durch schriftliche Stimmabgabe über:

a) Anträge, die die Verwaltung von sich aus durch die Gesamtheit der Mitglieder entscheiden lassen will;

b) Anträge, die durch Beschluss der Generalversammlung der Urabstimmung unterstellt werden.

\section{B. Delegiertenversammlung} Gestrichen

\section{Verwaltung}

\section{Art. 16 Zusammensetzung}

Die Verwaltung besteht aus dem Präsidenten und dem Generalsekretär der Verbindung der Schweizer Ärzte sowie aus zwei von der Generalversammlung oder durch Urabstimmung gewählten Personen, von denen beide Genossenschafter sein müssen. Der Präsident der Verbindung der Schweizer Ärzte ist ex officio auch Präsident des "FMH Services». Der Geschäftsleiter nimmt an den Sitzungen mit beratender Stimme teil. Er hat das Recht, Anträge zu stellen.

\section{* Die vollständigen Statuten können auf dem Internet unter www.fmhservices.ch eingesehen werden.}

Art. 4 Aufnahme

${ }^{1}$ Wer als

- ordentliches Mitglied oder als

- ausserordentliches Mitglied gemäss Statuten der Verbindung der Schweizer Ärztinnen und Ärzte FMH

in die Verbindung aufgenommen wird, kann gleichzeitig die Mitgliedschaft beim "FMH Services» beantragen.

${ }^{2}$ Mit der Beitrittserklärung zur Aufnahme in die Verbindung kann der Bewerber zugleich sein Einverständnis zum Beitritt in den «FMH Services» erklären.

\section{Stimmzettel}

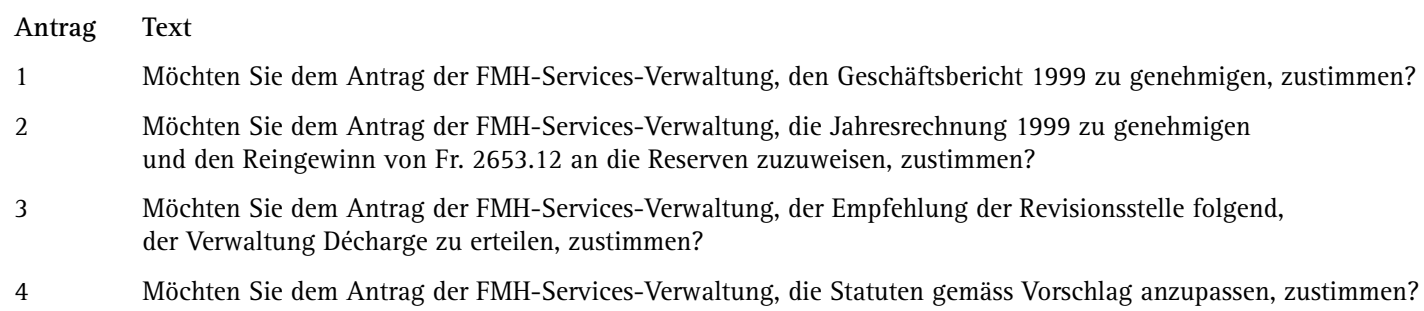




\section{Votation par correspon- dance FMH Services}

\begin{abstract}
Pourquoi une votation par correspondance?
En suite du changement des statuts de la Fédération des Médecins Suisses la qualité d'associé de la société coopérative FMH Services n'est plus conférée automatiquement aux membres FMH. Dès lors chaque membre FMH doit présenter une déclaration écrite afin d'acquérir la qualité d'associé. L'assemblée générale des délégués de la société FMH Services ne peut désormais plus être convoquée en même temps que le syndicat des médecins ordinaire puisque chaque membre du syndicat n'est pas nécessairement un associé de la société. Aussi l'administration a décidé de soumettre les affaires ordinaires et la modification des statuts nécessaire à une votation par correspondance.
\end{abstract}

\section{Qui a le droit de vote?}

Ont le droit de vote tous les membres FMH qui ont été admis comme membres ordinaires ou extraordinaires avant le 31.12.1999, ainsi que tous les membres FMH qui lors de leur entrée à la FMH ont souhaité d'acquérir aussi la qualité d'associé de la société FMH Services.

\section{Rapport de gestion 1999}

Les nouvelles structures de FMH Services ont été finalisées au cours de l'année passée. La société est maintenant structurée comme société holding. Chaque domaine opératoire est pris en charge par une société participante particulière. Il s'agit de sociétés anonymes indépendantes dont la majorité des actions restent avec la société holding. Le directeur de FMH Services est un membre du conseil d'administration de chaque société. Il informe l'administration sur les activités des sociétés particulières. Les informations détaillées au sujet des activités des sociétés de gestion sont présentées dans leurs rapports annuels qui sont également publiés dans le bulletin des médecins suisses. Ce regroupement en structure de holding permet une diminution de l'administration. Une administration ne consistant que de quatre au lieu de cinq personnes est capable d'accomplir ses tâches avec un plus haut degré de professionalité. L'admi-

nistration alors propose un changement des statuts à cet égard. Un changement des status sera également nécessaire puisque selon les nouveaux statuts de la Fédération des Médecins Suisses la qualité de membre de FMH et de FMH Services n'est plus automatiquement couplée. L'administration soumet les changements nécessaires à tous les membres sous forme de votation de correspondance.

Le bilan annuel de FMH Services reflète le changement des structures. Après la liquidation des réserves non dépensées qui résultaient dans un bénéfice de Fr. 244 000.- pour l'année précédente, le bilan annuel montre un bénéfice de Fr. 2635.-.

A la fin de l'année commerciale Dr Eduard Eicher, Bâle, va quitter l'administration. Les membres de l'administration et de la direction tiennent à le remercier pour son travail.

$\mathrm{Au}$ cours de l'année commerciale courante le groupe FMH Services va continuer à faire de son mieux pour agrandir sa palette de prestations en collaboration avec d'autres partenaires dans le domaine de santé publique et de mettre sa compétence à la disposition des membres. 


\section{Bilan au 31.12.1999}

Année commerciale 1999

Dénomination

Actifs

Capital de roulement

Banques

Titres

Total des disponibilités et titres

Créances

Créances envers des sociétés affiliées

Autres créances envers des sociétés affiliées

Autres créances

Total des créances

Actifs transitoires

Total du capital de roulement

Capitaux investi

Certificats de participation

Participation

Débiteurs à long terme

Total des immobilisations financières

Mobilier

Immeuble

Total du capital investi

Somme de Bilan
Fr. Dénomination

Fr.

Passifs

Capital dû aux tiers à court terme

107634.66

158263.20

265897.86

35890.45

12970.50

95016.62

3436.30

Créditeurs à court terme

Créditeurs à court terme, sociétés affiliées

Créditeurs à court terme, organisations affiliées

Passifs transitoires

Total du capital dû aux tiers à long terme

Capital dû aux tiers à long terme

Créditeurs à long terme

Provisions

147313.87

1215.25

414426.98

Total du capital dû aux tiers à long terme

Capital propre

Réserves

Bénéfices net

1000.00

617200.00

1620000.00

Total du capital propre

$\underline{\text { Somme de bilan }}$
36855.40

602.00

4706.10

20600.00

62763.50

745000.00

129750.00

874750.00

3008062.36

2653.12

3010715.48

3948228.98
2238200.00

63802.00

1231800.00

3533802.00

3948228.98

\section{Compte d'exploitation au 31.12.1999}

Année commerciale: 1999

Dénomination

Fr.

Total produit d'exploitation

Charges

Résultat brut 1

Frais de personnel

Résultat brut 2

Autres frais d'exploitation

Cash Flow

Amortissements

Résultat d'exploitation 1

Résultat d'exploitation annexes

Résultat d'exploitation 2

Résultat extraordinaire et hors exploitation

Résultat d'exploitation avant impôts

Impôts

Bénéfice net d'exploitation
1556.05

0.00

1559.15

$-52684.00$

$-51124.85$

$-37972.88$

$-89097.73$

$-60638.70$

$-149736.43$

124475.15

$-25261.28$

37991.20

12729.92

$-10076.80$

$\underline{2653.12}$

Rapport de l'organe de contrôle

L'organe de contrôle, la maison PriceWaterhouseCoopers, a vérifié la comptabilité et les comptes annuels et recommande d'approuver les comptes annuels. 


\section{Modification des statuts}

Plusieurs articles des statuts* de FMH Services sont à modifier. Les articles modifiés sont énumérés dans l'arrêté. Ensuite le texte des nouveaux articles est présenté.

\section{Arrêté}

Art. 31 Consentement de l'assemblée des délégués Ces statuts ont été acceptés par l'assemblée des délégués le 20 Juin 1990 et modifiés concernant Art. 1 le 20 juin 1996 et le 24 juin 1998.

\section{Art. 32 Modification des statuts par votation par} correspondance

Les status ont été modifiés lors de la votation par correspondance $\mathrm{du}$...... concernant:

II. Qualité d'associé, Art 4, alinéa 1 et 2

III. Organisation de "FMH Services», A. L'ensemble des associés, art. 9.b) et B. assemblée des délégués, art. 11 et art. 12, de même que C. administration, art. 16.

\section{Nouveau texte des articles mentionnés ci-dessus}

\section{Art. 4 Admission}

${ }^{1}$ Les personnes qui sont admis comme

- membres ordinaires ou

- membres extraordinaires de la Fédération des Médecins Suisses peuvent en même temps demander la qualité d'associé de FMH Services.

${ }^{2}$ En combinaison avec la déclaration d'entrée dans la Fédération les candidats peuvent consentir à entrer dans la société.

\section{A. L'Ensemble des membres}

\section{Art. 9 objet}

L'ensemble des membres décident en votant par correspondance sur

a) les propositions que l'administration désire soumettre à l'ensemble des membres;

b) les propositions qui suite à une décision de l'assemblée générale doivent être soumises à une votation par correspondance.

B. Assemblée des délégués Biffé

\section{Administration}

\section{Art. 16 Composition}

L'administration de la société se compose du président et du secrétaire général de la Fédération des Médecins Suisses de même que de deux personnes élues par l'assemblée générale ou lors d'une votation par correspondance; tous les deux doivent être des associés. Ex officio le président de la Fédération des Médecins Suisses sera également en fonction comme président de FMH Services. Le directeur participe aux discussions avec une voix consultative. Il a le droit de faire des propositions.

\footnotetext{
* Les statuts FMH Services sont publiés sur internet: www.fmhservices.ch
}

\section{Bulletin de vote}

$\begin{array}{ll}\text { Proposition } & \text { Texte } \\ 1 & \text { Acceptez-vous la proposition de l'administration de FMH Services d'approuver la rapport d'activité pour 1999? } \\ 2 & \begin{array}{l}\text { Acceptez-vous la proposition de l'administration de FMH Services d'approuver les comptes annuels pour } 1999 \\ \text { et d'attribuer le bénéfice net de Fr. 2653.12 aux réserves? }\end{array} \\ 3 & \begin{array}{l}\text { Acceptez-vous la proposition de FMH Services de suivre la recommandation de l'organe de révision } \\ \text { et de donner décharge à l'administration? }\end{array} \\ 4 & \text { Acceptez-vous la proposition de l'administration de FMH Services d'adapter les statuts selon la modification proposée? }\end{array}$

